\title{
Development and Implementation of a Facebook-Based Peer-to-Peer Support Group for Caregivers of Children With Complex Care Needs in New Brunswick
}

\author{
Katherine J. Kelly ${ }^{1}, \mathrm{MA}$; Alison Luke ${ }^{1}, \mathrm{PhD}$; and Shelley Doucet ${ }^{1}, \mathrm{PhD}$ \\ ${ }^{1}$ Department of Nursing and Health Sciences, University of New Brunswick \\ https://doi.org/10.15273/hpj.v1i1.10644
}

\begin{abstract}
Facebook has become an important gathering place for patients and caregivers to exchange healthrelated information and emotional support, otherwise known as peer-to-peer (P2P) support. Despite widespread use of Facebook groups across various patient and caregiver populations, the use of these groups by caregivers of children with complex care needs (CCCN) has not been previously reported. This paper describes the development and launch of a Facebook group for families of CCCN in New Brunswick, Canada, as well as the plans for evaluation and preliminary findings. The Facebook group was developed in consultation with various stakeholders, including a patient and family advisory council. The following factors were taken into consideration: group characteristics, moderators, language, recruitment, and implementation. The potential impact of the group on perceived knowledge of health services and/or resources and health literacy were assessed through semi-structured interviews with group members. The group, launched in October 2020, has been monitored for a period of 10 weeks for its use by caregivers. The group has attracted a total of 81 caregivers of CCCN, including two moderators. Inquiry-based posts were the most common type of posts made by members. The observed surge in group membership upon implementation suggests the need for additional P2P support platforms for caregivers of CCCN in New Brunswick. Ongoing monitoring and evaluation will determine how the group is used by members and whether it has any effect on health literacy and knowledge of resources and services.
\end{abstract}

Keywords: peer-to-peer support, social support, social media, children with health care needs 
Development and Implementation of a Facebook-Based Peer-to-Peer Support Group for Caregivers of Children With Complex Care Needs in New Brunswick

Caregivers of children with complex care needs (CCCN) often have multiple unmet informational needs and require timely access to services and resources to ensure the wellbeing of their families (Roche \& Skinner, 2009). Accessing this support can be challenging for families that encounter barriers, such as access to primary care or financial aid (Charlton et al., 2017), particularly during the COVID-19 pandemic. Online peer-to-peer (P2P) support groups, defined as online communities of individuals with common lived experiences (Naslund et al., 2016), provide an innovative means of connecting and supporting families. Caregivers of CCCN possess invaluable lived experience and knowledge related to available and effective services and programs; connecting these families together through P2P support has the potential to promote information sharing among a community of patients and caregivers in a safe, secure online environment.

Current evidence on the use of social media-based P2P support groups has relied on content analysis of posts from publicly accessible groups, primarily those of patients with various health concerns (Bender et al., 2011; Farmer et al., 2009). This paper describes the development and evaluation plan of a geographic-specific P2P support group for caregivers of CCCN in New Brunswick (NB), Canada. Facebook will be used as the platform for this group. In this study, CCCN are defined as children with multi-dimensional health and social care needs, who may or may not possess a diagnosis of a recognized condition (Brenner et al., 2018).

\section{Background and Rationale}

Children with CCCN present across diverse settings, requiring services from multiple care providers, which can result in significant physical, mental, and emotional pressures on caregivers. These pressures have been further exacerbated by the COVID-19 pandemic, due to increased strain on health and social services (World Health Organization, 2020). Social distancing measures, for example, have led to unique challenges for caregivers of CCCN, leading to increased caregiver stress and loneliness (American Psychological Association, 2020).

Online P2P support offers an accessible and inexpensive source of informational knowledge and emotional support (Rossman, 2007). P2P groups have been shown to provide individuals with valuable and timely informational, social, and emotional support without participants leaving their homes (Cole et al., 2017; Diefenbeck et al., 2017; Partridge et al., 2018). These benefits have been observed in groups of individuals with chronic disease (Partridge et al., 2018), multiple sclerosis (Shavazi et al., 2016), and HIV (Henwood et al., 2016), and caregivers of individuals with chronic illnesses (Diefenbeck et al., 2017; Scharett et al., 2017). The relationships formed through online interactions can result in feelings of community and solidarity (Horter et al., 2014), which can influence a sense of belonging (Anderson \& Emmerton, 2016). This is an important consideration for families of CCCN in NB, who have reported feeling socially disconnected from resources that help them meet their child's care needs (Charlton et al., 2017).

Studies focusing on P2P support groups on social media have noted the widespread use of Facebook groups across different types of patient and caregiver populations (Bender et al. 2011, Farmer et al., 2009; Naslund et al., 2016), including families of CCCN. In an exploratory study on the uses of online groups by 18 parents of CCCN, Ammari et al. (2014) noted that caregivers primarily used Facebook for accessing informational and social support. Specifically, parents accessed geographicspecific groups for locally-based support and case-based groups for support related to specific conditions. These findings suggest that geographic-based communities can meet the support needs of caregivers of CCCN; however, it remains unclear how caregivers of CCCN use 
these geographic-specific support groups and what potential impact that membership might have on meeting the support needs of caregivers. Leveraging existing social media to explore the use of P2P support groups by caregivers of CCCN can provide important insight into the culture of online information sharing and emotional support and how it can benefit and meet the needs of caregivers.

For many individuals, the internet is a critical resource in searching for and locating services, programs, and resources. P2P support can increase knowledge of health-related resources and services (Santelli et al., 1997), which can, in turn, affect health literacy. Health literacy is typically defined as an individual's ability to read and understand health information (Powers et al., 2010). Health literacy is negatively associated with health inequalities, which are impacted by a person's living and material circumstances, as well as socio-economic status (Harris et al., 2015). Improved access to information about available health and social programs can empower caregivers and provide them with confidence when interacting with the Canadian health care system (Jackson et al., 2019). However, it is unclear whether social media-based P2P support can improve knowledge of healthrelated services and programs of caregivers of CCCN. There is evidence, however, that low caregiver health literacy has been linked to difficulties in navigating the health care system, which can result in fragmentation of care (Fields et al., 2018); this link between low health literacy and patient care has also been observed in parents/guardians caring for a child (Lee et al., 2014).

Despite the benefits that Facebookbased P2P support groups can provide for caregivers, ensuring the retention and success of these groups can be difficult for administrators. According to internal data from Facebook, approximately 100,000 new groups are created every day; however, less than half of these groups (43\%) survive past the first three months (Kraut \& Fiore, 2014). Research on general Facebook groups has identified specific variables that promote short-term success, such as presence of moderators (Kraut \& Fiore, 2014), membership size (La Macchia et al., 2016), privacy (Ma et al., 2019), and established rules (Moser et al., 2017). For example, group administrators can promote the initial success of P2P support groups by posting content and frequently interacting with posts by other members (Booth, 2012; Kraut \& Fiore, 2014). However, it is unclear what variables might lead to the overall sustainability of these groups.

Online P2P support groups present an opportunity for caregivers of CCCN to learn about and make sense of the maze of services, programs, and treatments available to them, as well as the overwhelming amount of information provided to them from various sources (DeHoff et al., 2016). It gives families control and presents a safe environment in which to exchange emotional support with peers (Zhao \& Zhang, 2017). Facebook, in particular, has been noted as an important gathering place for individuals with health care needs and their caregivers (Farmer et al., 2009); however, there currently exist no active P2P support groups for caregivers of CCCN in NB on Facebook (Kelly et al., 2020). Previous research has suggested that caregivers of CCCN prefer to connect with other caregivers who have similar experiences and live in their geographic area (Ammari et al., 2014; Scharer, 2005); however, the use and perceived benefits of such groups by caregivers of CCCN is currently unknown. Specifically, it is unclear whether a geographicspecific Facebook P2P support group can improve knowledge of health-related services and resources and increase sense of social belonging in caregivers of CCCN. This paper describes the development, launch, and preliminary observations of a Facebook P2P support group for caregivers of CCCN in NB and outlines the plan for evaluation.

\section{Study Objectives and Research Questions}

The current research sought to investigate the use of a Facebook-based P2P support group by caregivers of CCCN in NB. This research consisted of three steps: (a) develop a Facebook P2P support group for caregivers of CCCN, (b) assess its use by caregivers of CCCN, 
and (c) explore potential impacts on knowledge of health services and/or resources and sense of social belonging among caregivers of CCCN. The first section of this paper will describe the development and launch of the Facebook P2P support group. The second section will outline the protocol for evaluation and outline preliminary observations.

\section{Primary Objectives}

The primary objective in this research was to develop and implement a geographic-specific Facebook P2P support group, in collaboration with a patient and family advisory council, and investigate its use and perceived benefits by caregivers of CCCN. The following research questions form the basis for this research:

1. How is the P2P support Facebook group used by NB caregivers of CCCN?

2. What are the experiences of caregivers of CCCN who use the Facebook group to communicate with other caregivers of CCCN?

3. In what ways does participating in the Facebook group affect NB caregivers of CCCNs' perceived knowledge of services or resources and sense of belonging?

\section{Secondary Objectives}

The secondary objective of this research is to better understand factors that relate to the use of the group. Success of the group will be determined by a consistent level of interaction between members of the group. The following research question was used to explore this objective:

4. What factors contribute to the success or failure of the Facebook group for caregivers of CCCN in NB?

\section{Facebook Group Development}

A P2P support group was developed using the Facebook platform, which is a free social media website. The group was developed in response to a needs assessment that found that NB families of CCCN feel disconnected from local resources (Charlton et al., 2017), and an environmental scan which revealed that there are currently no P2P support groups on Facebook for families of CCCN in NB (Kelly et al., 2020). The Facebook group, developed for the purpose of this research, is restricted to screened members who live in NB. The purpose of this group is to facilitate the exchange of informational and emotional support between caregivers of CCCN.

The group was developed in consultation with a team composed of stakeholders from NaviCare/SoinsNavi (NB's patient navigation centre for families of CCCN), the University of New Brunswick, Mount Allison University, and the University of Prince Edward Island. Focus groups were held with NaviCare/SoinsNavi's Patient and Family Advisory Council (PFAC), which is composed of six caregivers of CCCN and one young adult who grew up with complex care needs. Group components were vetted by the PFAC, including the title, description, and membership requirements. Table 1 below presents the final iteration of details published on the group.

\section{Membership Screening}

Prospective members are required to undergo a short screening prior to gaining admittance to the Facebook group. This screening consists of two short questions presented to the user when they request to join the group:

1. Do you identify as a caregiver of a child or youth with health care need(s)?

2. Do you live in New Brunswick, Canada?

An additional required question asks prospective members to type "I consent". This question provides users with an explanation of the purpose of the group from a research perspective and provides links to the letter of information and informed consent form:

3. CONSENT REQUIRED: Please read the page at the following link and type "I consent" after reading: https://bit.ly/3mHPSeY 
Table 1

Facebook Group Final Components

\begin{tabular}{|c|c|}
\hline Component & Description of Component Details \\
\hline Title & NB Children with Health Care Needs \\
\hline Description & $\begin{array}{l}\text { This group is for parents, guardians, and other caregivers of children and } \\
\text { youth with health care needs in New Brunswick. This is a safe place to } \\
\text { connect with other NB families and share/learn about local services and } \\
\text { resources. It is also a place to find answers to your questions and to help } \\
\text { others who are on a similar journey. We welcome anyone who cares for a } \\
\text { child/youth with any health care need(s). } \\
\text { Please note that this group is developed and managed by } \\
\text { NaviCare/SoinsNavi (a research-based patient navigation centre based in } \\
\text { New Brunswick) to facilitate the exchange of services and resources } \\
\text { between caregivers (e.g., parents and guardians) of children and youth with } \\
\text { health care needs in NB. This Facebook group serves as a pilot project to } \\
\text { better understand online peer-to-peer support. This means that the group } \\
\text { is monitored for use and members will be invited to participate in a } \\
\text { research interview; however, the information shared within this group will } \\
\text { never be made publicly available or shared outside of the group. This } \\
\text { project has been approved by the Research Ethics Board at UNB. The letter } \\
\text { of information and informed consent is available at the following } \\
\text { link: https://bit.ly/3mHPSeY. By participating in this group and answering } \\
\text { the third membership question, you are agreeing to participate in this } \\
\text { study. }\end{array}$ \\
\hline Privacy designation & Closed group \\
\hline Language & Bilingual \\
\hline Moderators & NaviCare/SoinsNavi's PFAC members and patient navigators \\
\hline
\end{tabular}

Note. French translation of the title and description is provided in the group. 


\section{Moderators}

The use of moderators was posited to be an important contribution to the activity levels (i.e., success) and long-term sustainability of online P2P support groups (Biagianti et al., 2018). Group moderators currently consist of the NaviCare/SoinsNavi patient navigator, who is a Registered Nurse, and a member of the PFAC. Moderators conduct the following tasks on a daily basis: screen prospective members, monitor ongoing discussions, and provide responses to posts without replies.

The patient navigator provides a novel contribution as a health professional in a $\mathrm{P} 2 \mathrm{P}$ support group; however, her role within the group is simply to offer one perspective. The lived experiences of other caregivers provide a rich dialogue and exchange of social support that is only available from someone on a similar journey.

\section{Implementation and Recruitment Strategy}

After details related to the group were vetted and approved by NaviCare/SoinsNavi's PFAC, the Facebook group was officially launched on October 5, 2020. Members of the PFAC and research team were invited to review the final iteration of the group. Recruitment of group members started on October 13, 2020, using the following strategies:

- invitation to caregivers enrolled in NaviCare/SoinsNavi, sent by their patient navigator;

- media release to 35 community organizations that support families of CCCN in NB;

- $\quad$ posts made on existing Facebook groups and pages used by families in NB (e.g., car seat safety group, etc); and

- media releases to various digital newsletters and online boards off the Facebook platform.

This recruitment strategy was completed on October 20, 2020. Ongoing recruitment primarily consists of word-ofmouth from group members and advertisement of the group on NaviCare/SoinsNavi's public Facebook page.

\section{Evaluation Plan}

\section{Study Design}

The Facebook group will be evaluated using a qualitative descriptive design to understand the perceived impact and use of a Facebook-based P2P support group for caregivers of CCCN. Qualitative description is a pragmatic qualitative approach that facilitates obtaining straightforward answers to questions in applied health research (Sandelowski, 2000). A qualitative model was chosen to explore the uses and benefits of a Facebook-based P2P support group absent of preconceived models or restrictions.

\section{Study Population}

All members of the Facebook group represent the study population. An online survey and semi-structured interviews will take place with a subset of volunteers from within the group. A survey will be distributed to members through a post on the group's discussion board; participants will be asked at the conclusion of the survey if they are interested in being contacted for an interview. Interview participants are required to have been in the group for a minimum of three months; this length of time is specified to ensure that members have had time to interact with content and members within the group. A total of 50 survey participants and 20-25 individual interviews will be sought beginning in January 2021. These numbers were chosen to ensure membership representation and information saturation.

\section{Data Collection}

Group members will be invited to take part in an online survey and interview about their use and experience as a member of the group (Research Questions \#1 and \#2) and perceived knowledge of health services and resources (Research Question \#3). The online survey consists of 15 closed-ended questions (e.g., "Have you learned about any services or resources for children/youth with health care needs in New Brunswick as a result of your membership in NB Children with Health Care 
Needs?") and three open-ended questions (e.g., "In what ways has the NB Children with Health Care Needs Facebook group helped you, or can help you, address some of your needs in your role as a caregiver?"). Interview questions were structured based on users' experience of the P2P support group (e.g., "In what ways did you or did you not find that the Facebook group was able to meet your needs or provide needed support?") and its effect on accessibility and knowledge of the health care system (e.g., "How did the P2P support group affect your knowledge of existing health-related resources or services?").

The online survey was created using Qualtrics XM. Interviews will take place using Zoom video conferencing software, using the steps outlined by Lobe et al. (2020) regarding social distancing interview measures, given the current pandemic situation. Zoom was chosen due to previous empirical support for its ease of qualitative data collection, data management features, and security options (Archibald et al., 2019). Content published to the group (e.g., posts, replies, etc) will be regularly collected and organized into Excel to better understand the use of the group by members and moderators (Research Question \#1). Specifically, Facebook posts will be labelled according to one of six categories based on the information provided in the post: information, emotional, inquiry, advertising, fundraising, and other (Bender et al., 2011; Farmer et al., 2009). Information posts are those containing information of relevance (e.g., shared academic article or news post). Emotional posts describe posts relating an experience, story, or narrative, often allowing a user to share frustrations or successes. Inquiry posts contain a question or set of inquiries from members; this type of post differs from information posts, in that it is primarily based around a question. Advertising posts include a notice regarding an event or sale of a product, and fundraising posts aim to raise funds for a particular cause. Finally, posts designated as "other" are any posts that do not fit one of the previous five categories.

Factors related to the success or failure of the proposed P2P support group, as determined by membership activity, will be regularly collected throughout the evaluation period (Research Question \#4). Specifically, the type of posts (as described above) and associated interactions (i.e., likes and comments by members), as well as date and time of post will be counted and kept in an Excel file on a weekly basis. Other indicators suggested in the literature to promote or hinder activity by members, such as number of administrators, involvement of moderators in discussions, and membership size (Biagianti et al., 2018; Kraut \& Fiore, 2014), will be recorded throughout the course of the study period. These factors will be compared to the number of published posts, and corresponding interactions, to determine whether there is an association between changes to the group and higher or lower activity levels. Outcomes will be collected throughout the study period (Fall 2020-Spring 2021) and will be analyzed at the conclusion of the study (Spring 2021).

\section{Data Analysis}

Survey results from closed-ended questions will be analyzed in Microsoft Excel. Interviews will be recorded using a digital voice recorder and then transcribed verbatim and managed using NVivo software (version 1.0). Open-ended survey questions and interview transcripts will be analyzed using thematic analysis (Braun \& Clarke, 2006; Willis et al., 2016). Content collected from the P2P support group (i.e., posts and replies) will be analyzed using content analysis. Content analysis differs from thematic analysis in that it aims to provide a mixed methods approach to describing a phenomenon (i.e., qualitative coding and use of quantitative counts), whereas thematic analysis provides a detailed and in-depth description of qualitative data (Braun \& Clarke, 2006; Vaismoradi et al., 2013).

\section{Risk Mitigation}

A variety of risk mitigation strategies associated with the use of online P2P support forums have been identified within the literature. These include monitoring discussion posts to prevent the spread of misinformation, lack of replies, technical problems, and data 
security (Niela-Vilén et al., 2014). Discussion moderators monitor discussion boards to respond to posts and ensure the validity of information on a regular basis. Moderators are trained using best practice protocols (Schippke et al., 2015), adhering to a guide developed specifically for this project. Specifically, all members are provided with clear rules for participating in the P2P support group, which are enforced by moderators. Members who fail to adhere to rules are asked by a moderator to review the group rules and warned that if they continue to break the rules, their membership will be revoked. Misinformation is clarified in a timely manner by moderators, by providing correct information with appropriate source(s).

\section{Preliminary Observations}

At the time of writing this paper (December 2020), the Facebook group (NB Children with Health Care Needs) has been live for a period of 10 weeks. The group has a total of 81 members, two of whom act as discussion moderators. Prior to the development and launch of this group, there were no P2P support groups available on the platform for NB families (Kelly et al., 2020). However, it has become clear that there is a need for such a group by the immediate surge in membership after the launch of the group.

The first six weeks of the group represented a period of intense growth in membership, but little interaction between members on the discussion board. A social media plan was put together by moderators to encourage interaction by members; this resulted in weekly discussion posts that prompt members to answer various questions. Moderators posited that members might have been uncomfortable being the first to post; therefore they reached out to a member of the group to ask if she might be comfortable posting a question. This led to a surge in novel posts by members, predominately inquiry-type posts related to caring for a child during the COVID-19 pandemic and locating resources to support their child. Other types of posts published in the group have largely consisted of shared resources from other pages, such as information about CCCN webinars or information about developmental assessments or therapy. The increase in membership activity levels (i.e., posting and interaction) observed in response to weekly discussion posts by the moderators suggests that pre-existing posts may encourage content publication from existing and new members (Booth, 2012), in turn promoting group success.

At this time, a total of 45 posts have been made in the Facebook group. A total of 17 $(37.7 \%)$ of these posts were categorized as inquiry-based posts. A further 14 were classified as "other" (e.g., administration update); 11 were information-based posts (e.g., post on an upcoming webinar of relevance); and two were fundraising-based posts.

The moderating team meets on a weekly basis to discuss activity within the group and any concerns that arise. The lead researcher (KK) meets with NaviCare/SoinsNavi's PFAC on a monthly basis to present updates on the group and receive feedback. This feedback consists of ways to promote group interaction and content, as well as input on the research methods and analysis of the project. Determining the factors that affect interaction between members and ensure the longevity of the group continues to be a challenge in the infancy stage of this group due to lack of data; however, the increase in membership activity level is expected to provide further insight into these factors over time. The online survey will be distributed to members in early January 2021, and interviews will begin in February 2021.

\section{Conclusion}

This research aims to explore the use of a Facebook-based P2P support group by caregivers of CCCN in NB. The positive response to the group, as indicated by a surge in membership within only four weeks, suggests a possible need for better support and interaction among caregivers of CCCN in NB. Ongoing monitoring and evaluation will determine how the group is used by members and whether it 
has any effect on health literacy and knowledge of resources and services.

\section{Author Note}

Katherine J. Kelly, https://orcid.org/0000-0001-5405-255X

We have no known conflict of interest to disclose. Correspondence concerning this article should be addressed to Katherine J. Kelly, Interdisciplinary studies, kj.kelly@unb.ca

\section{References}

American Psychological Association. (2020, March). Advice for caregivers of children with disabilities in the era of COVID-19. https://www.apa.org/research/action/ children-disabilities-covid-19

Ammari, T., Schoenebeck, S. Y., \& Morris, M. R. (2014). Accessing social support and overcoming judgment on social media among parents of children with special needs. In Proceedings of the Eighth International AAAI Conference on Weblogs and Social Media (pp. 22-31). The AAAI Press.

Anderson, K., \& Emmerton, L. M. (2016). Contribution of mobile health applications to self-management by consumers: Review of published evidence. Australian Health Review, 40(5), 591-597. c8h. https://doi.org/10.1071/AH15162

Archibald, M. M., Ambagtsheer, R. C., Casey, M. G., \& Lawless, M. (2019). Using Zoom videoconferencing for qualitative data collection: Perceptions and experiences of researchers and participants. International Journal of Qualitative Methods, 18. https://doi.org/10.1177/16094069198 74596

Bender, J. L., Jimenez-Marroquin, M.-C., \& Jadad, A. R. (2011). Seeking support on Facebook: A content analysis of breast cancer groups. Journal of Medical
Internet Research, 13(1), Article e16. https://doi.org/10.2196/jmir.1560

Biagianti, B., Quraishi, S. H., \& Schlosser, D. A. (2018). Potential benefits of incorporating peer-to-peer interactions into digital interventions for psychotic disorders: A systemic review. Psychiatric Services, 69(4), 377-388. https://doi.org/10.1176/appi.ps.20170 0283

Booth, S. E. (2012). Cultivating knowledge sharing and trust in online communities for educators. Journal of Educational Computing Research, 47(1), 1-31. https://doi.org/10.2190\%2FEC.47.1.a

Braun, V., \& Clarke, V. (2006). Using thematic analysis in psychology. Qualitative Research in Psychology, 3(2), 77-101.

Brenner, M., O’Shea, M. P., McHugh, R., Clancy, A., Larkin, P., \& Luzi, D. (2018). Principles for provision of integrated complex care for children across the acute-community interface in Europe. The Lancet: Child \& Adolescent Health, 2(11), 832-838. https://doi.org/10.1016/S23524642(18)30270-0

Charlton, P., Azar, R., Luke, A., Doucet, S., Montelpare, W., Nagel, D., Hyndman, N., \& Thompson, K. (2017). Falling through the cracks: Barriers to accessing services for children with complex health conditions and their families in New Brunswick. Journal of New Brunswick Studies, 8, 133-158.

Cole, H., Thompson, H. S., White, M., Browne, R., Trinh-Shevrin, C., Braithwaite, S., Fiscella, K., Boutin-Foster, C., \& Ravenell, J. (2017). Community-based, preclinical patient navigation for colorectal cancer screening among older Black men recruited from barbershops: The MISTER B Trial. American Journal of Public Health, 107(9), 1433-1440. https://doi.org/10.2105/AJPH.2017.30 3885

DeHoff, B. A., Staten, L. K., Rodgers, R. C., \& Denne, S. C. (2016). The role of online 
social support in supporting and educating parents of young children with special health care needs in the United States: A scoping review. Journal of Medical Internet Research, 18(12), Article e333. https://doi.org/10.2196/jmir.6722

Diefenbeck, C. A., Klemm, P. R., \& Hayes, E. R. (2017). "Anonymous meltdown": Content themes emerging in a nonfacilitated, peer-only, unstructured, asynchronous online support group for family caregivers. CIN: Computers, Informatics, Nursing, 35(12), 630-638. https://doi.org/10.1097/CIN.00000000 00000376

Farmer, A. D., Bruckner Holt, C. E. M., Cook, M. J., \& Hearing, S. D. (2009). Social networking sites: A novel portal for communication. Postgraduate Medical Journal, 85(1007), 455-459. https://doi.org/10.1136/pgmj.2008.07 4674

Fields, B., Rodakowski, J., James, A. E., \& Beach, S. (2018). Caregiver health literacy predicting healthcare communication and system navigation difficulty. Families, Systems, and Health, 36(4), 482-492. https://doi.org/10.1037/fsh0000368

Harris, J., Springett, J., Croot, L., Booth, A., Campbell, F., Thompson, J., Goyder, E., Van Cleemput, P., Wilkins, E., \& Yang, Y. (2015). Can community-based peer support promote health literacy and reduce inequalities? A realist review. Public Health Research, 3(3). https://doi.org/10.3310/phr03030

Henwood, R., Patten, G., Barnett, W., Hwang, B., Metcalf, C., Hacking, D., \& Wilkinson, L. (2016). Acceptability and use of a virtual support group for HIV-positive youth in Khayelitsha, Cape Town using the MXit social networking platform. AIDS Care, 28(7), 898-903. https://doi.org/10.1080/09540121.20 16.1173638

Horter, S., Stringer, B., Venis, S., \& du Cros, P. (2014). "I can also serve as an inspiration": A qualitative study of the TB\&Me blogging experience and its role in MDR-TB treatment. PLoS ONE, 9(9), Article e108591.

https://doi.org/10.1371/journal.pone.0 108591

Jackson, C., Snyder, J., Crooks, V. A., \& Lavergne, M. R. (2019). Exploring isolation, selfdirected care and extensive follow-up: Factors heightening the health and safety risks of bariatric surgery abroad among Canadian medical tourists. International Journal of Qualitative Studies on Health and Well-being, 14(1), Article 1613874. https://doi.org/10.1080/17482631.20 19.1613874

Kelly, K., Doucet, S., Luke, A., Azar, R., \& Montelpare, W. (2020). Peer-to-peer support on Facebook for families of children with complex care needs in an Atlantic Canadian Province: An environmental scan [Manuscript submitted for publication]. Department of Nursing and Health Sciences, University of New Brunswick.

Kraut, R. E., \& Fiore, A. T. (2014). The role of founders in building online groups. In CSCW '14: Proceedings of the 17th ACM Conference on Computer Supported Cooperative Work \& Social Computing (pp. 722-732). Association for Computing Machinary. https://doi.org/10.1145/2531602.253 1648

La Macchia, S. T., Louis, W. R., Hornsey, M. J., \& Leonardelli, G. J. (2016). In small we trust: Lay theories about small and large groups. Personality and Social Psychology Bulletin, 42(10), 1321-1334. https://doi.org/10.1177/01461672166 57360

Lee, Y. J., Boden-Albala, B., Larson, E., Wilcox, A., \& Bakken, S. (2014). Online health information seeking behaviors of Hispanics in New York City: A community-based cross-sectional study. Journal of Medical Internet Research, 
16(7), Article e176. https://doi.org/10.2196/jmir.3499

Lobe, B., Morgan, D., \& Hoffman, K. A. (2020).

Qualitative data collection in an era of social distancing. International Journal of Qualitative Methods, 19. https://doi.org/10.1177/16094069209 37875

Ma, X., Cheng, J., Iyer, S., \& Naaman, M. (2019). When do people trust their social groups? In CHI '19: Proceedings of the 2019 CHI Conference on Human Factors in Computing Systems (Paper No. 67). Association for Computing Machinery. https://doi.org/10.1145/3290605.330 0297

Moser, C., Resnick, P., \& Schoenebeck, S. (2017). Community commerce: Facilitating trust in mom-to-mom sale groups on Facebook. In CHI '17: Proceedings of the 2017 CHI Conference on Human Factors in Computing Systems (4344-4357). Association for Computing Machinery. https://doi.org/10.1145/3025453.302 5550

Naslund, J. A., Aschbrenner, K. A., Marsch, L. A., \& Bartels, S. J. (2016). The future of mental health care: Peer-to-peer support and social media. Epidemiology and Psychiatric Sciences, 25(2), 113122.

https://doi.org/10.1017/S2045796015 001067

Niela-Vilén, H., Axelin, A., Salantera, S., \& Melender, H.-L. (2014). Internet-based peer support for parents: A systematic integrative review. International Journal of Nursing Studies, 51(11), 1524-1537. https://doi.org/10.1016/j.ijnurstu.201 4.06.009

Partridge, S. R., Gallagher, P., Freeman, B., \& Gallagher, R. (2018). Facebook groups for the management of chronic diseases. Journal of Medical Internet Research, 20(1), Article e21. https://doi.org/10.2196/jmir.7558

Powers, B. J., Trinh, J. V., \& Bosworth, H. B. (2010). Can this patient read and understand written health information?
Journal of the American Medical Association, 304(1), 76-84. https://doi.org/10.1001/jama.2010.89 6

Roche, M. I., \& Skinner, D. (2009). How parents search, interpret, and evaluate genetic information obtained from the internet. Journal of Genetic Counseling, 18(2), 119-129. https://doi.org/10.1007/s10897-0089198-4

Rossman, B. (2007). Breastfeeding peer counselors in the United States: Helping to build a culture and transition of breastfeeding. Journal of Midwifery \& Women's Health, 52(6), 631-637. https://doi.org/10.1016/j.jmwh.2007.0 5.006

Sandelowski, M. (2000). Focus on research methods: Whatever happened to qualitative description? Research in Nursing \& Health, 23(4), 334-340. https://doi.org/10.1002/1098240X(200008)23:4\%3C334::AIDNUR9\%3E3.0.CO;2-G

Santelli, B., Turnbull, A., \& Higgins, C. (1997). Parent to parent support and health care. Pediatric Nursing, 23(3), 303-306.

Scharer, K. (2005). Internet social support for parents: The state of science. Journal of Child and Adolescent Psychiatric Nursing, 18(1), 26-35. https://doi.org/10.1111/j.17446171.2005.00007.x

Scharett, E., Madathil, K. C., Lopes, S., Rogers, H., Agnisarman, S., Narasimha, S., Ashok, A., \& Dye, C. (2017). An investigation of the information sought by caregivers of Alzheimer's patients on online peer support groups. Cyberpsychology, Behavior, and Social Networking, 20(10), 640-657. https://doi.org/10.1089/cyber.2017.02 74

Schippke, J., Provvidenza, C., \& Kingsnorth, S. (2015). Rapid evidence review: Peer support for Ontario families of children with disabilities. Evidence to Care, Holland Bloorview Kids Rehabilitation 


\begin{tabular}{|l|l|l}
\hline 1 & HEALTHY \\
POPULATIONS \\
JOURNAL
\end{tabular}

Hospital.

https://hollandbloorview.ca/sites/defa

ult/files/2019-

06/EtC_PeerSupport_RER_FA.pdf

Shavazi, M. A., Morowatisharifabad, M. A.,

Shavazi, M. T. A., Mirzaei, M., \& Mellat

Ardekani, A. (2016). Online social

support for patients with multiple

sclerosis: A thematic analysis of

messages posted to a virtual support

community. International Journal of

Community Based Nursing and

Midwifery, 4(3), 188-198.

Vaismoradi, M., Turunen, H., \& Bondas, T.

(2013). Content analysis and thematic analysis: Implications for conducting a qualitative descriptive study. Nursing \& Health Sciences, 15(3), 398-405. https://doi.org/10.1111/nhs.12048

Willis, D. G., Sullivan-Bolyai, S., Knafl, K., \&

Cohen, M. Z. (2016). Distinguishing features and similarities between descriptive phenomenological and qualitative description research. Western Journal of Nursing Research, 38(9), 1185-1204.

https://doi.org/10.1177/01939459166 45499

World Health Organization. (2020, June 1).

Maintaining essential health services: Operational guidance for the COVID-19 context, interim guidance (Document No. WHO/2019-

nCoV/essential_health_services/2020.). https://apps.who.int/iris/bitstream/ha ndle/10665/332240/WHO-2019-nCoVessential_health_services-2020.2eng.pdf?sequence $=1$ \&isAllowed $=y$

Zhao, Y., \& Zhang, J. (2017). Consumer health information seeking in social media: A literature review. Health Information and Libraries Journal, 34(4), 268-283. https://doi.org/10.1111/hir.12192 